Topiques, études satoriennes

Topoï Studies, Journal of the SATOR

\title{
Appropriation de la modernité, retour à l'antique et inversion de la relation de maître à disciple : Diderot entre Eschyle et Updike
}

\section{Michel-Guy Gouverneur}

Volume 4, 2018

Maître-disciple : une relation topique

URI: https://id.erudit.org/iderudit/1074724ar

DOI: https://doi.org/10.7202/1074724ar

See table of contents

Publisher(s)

SATOR, Société d'Analyse de la Topique Romanesque d'Ancien Régime

ISSN

2369-4831 (digital)

Explore this journal

Cite this article

Gouverneur, M.-G. (2018). Appropriation de la modernité, retour à l'antique et inversion de la relation de maitre à disciple : Diderot entre Eschyle et Updike. Topiques, études satoriennes / Topoï Studies, Journal of the SATOR, 4, 1-21. https://doi.org/10.7202/1074724ar

\section{Article abstract}

From (M/m)entor to master or guide, the function of the tutor/trainer has been mapped by various authors in Western monarchies. One man questioned this apparent consensus in XVIIIth c. France : Diderot 's evolution in this respect is striking and the reason why his position shifted is clearly related to his discovering the character of Prometheus in the Greek text. Hie may still be found to have post-modern followers, even in contemporary America.
This document is protected by copyright law. Use of the services of Érudit (including reproduction) is subject to its terms and conditions, which can be viewed online.

https://apropos.erudit.org/en/users/policy-on-use/ 
Michel-Guy Gouverneur

\section{Appropriation de la modernité, retour à l'antique et inversion de la relation de maître à disciple : Diderot entre Eschyle et Updike.}

\section{Introduction}

Dès qu'une civilisation se comprend comme telle, la question de la transmission (que transmettre et comment le transmettre) occupe le premier plan; Athènes a connu la $\pi \alpha 1 \delta \varepsilon i ́ \alpha$ qui finit par acquérir une telle importance qu'elle s'identifiait, dès l'époque hellénistique à ce que nous appelons aujourd'hui la culture. Le retour à l'antique qui anime l'humanisme européen à la Renaissance n'est sans doute pas étranger à la façon dont les Rabelais, Montaigne, et plus tard Molière ou Fontenelle posent la question du sujet au risque de l'apprentissage : l'éducation opérée par le monde instruit-elle, construit-elle, ou détruit-elle l'enfant ? Se pose alors la question du précepteur (qu'il soit initiateur de bien plutôt qu'érudit) puis du mentor (qu'il incite le disciple à découvrir ses ressources au terme d'une expérience sagement guidée). Avec Fénelon, on atteint le point d'origine - l'antonomase du mentor en est preuve — d'un idéal d'éducation où les mesures du legs deviennent secondaires par rapport à l'âme de l'héritier.

Dans ce plan d'équilibre, l'anxiété, le génie créateur, de Diderot ont tôt fait de 'déconstruire' quelques principes tenus pour acquis, et d'ouvrir un nouveau champ de réflexion : la vérité humaine comme substitut de la sagesse, la dissociation étude / éducation, les apories de la transmission (désapprendre, émanciper, former ou informer ?), la réciprocité de l'apprentissage ..., c'est au travers de ce questionnement que Diderot conquiert sa part de modernité, et tout l'objet de la présente recherche est de faire apparaître les difficultés et les succès de son évolution. Je propose d'inverser la relation du mentorat, de s'intéresser au cas où le maître s'ignore comme tel, et où le disciple se présente comme désir, simple désir de se rattacher à un maître présumé. Les exemples que je prendrai seront des cas-limites dans la mesure où l'auteur étudié postule l'autonomie plus que l'autorité comme principe idéal de l'organisation sociale, et où le contexte dans lequel s'inscrit l'œuvre est aussi celui que menace le processus de dilution de l'Ancien Régime. 


\section{Un neveu sans éducation?}

Chez Fénelon, Télémaque incarne la jeunesse et surtout ses errements : l'inexpérience, l'irréflexion, l'imprudence, une forme d'égoïsme mais aussi une spontanéité qui porte à préférer la vérité au mensonge, le besoin de comprendre et d'apprendre, la curiosité d'esprit. Ce que veut montrer l'auteur dans ce cas, c'est le perfectionnement d'un disciple dans un parcours fait d'épreuves au travers desquelles il peut égaler son maître, même si ce dernier le dépasse toujours en sagesse. Mais tous deux auront gagné en humanité, l'un en s'approchant de son modèle, en acquérant de l'expérience, et en découvrant ses propres forces, l'autre en admettant les traces de l'imperfection humaine.

Par un jeu assez complexe et visionnaire sur les expériences parallèles et distinctes du lecteur-spectateur, de l'acteur-personnage et de l'auteur, Diderot enterre, avec Le Neveu de Rameau (1762-1773), la vision traditionnelle du mentor pour faire naître une figure moderne du modèle saisi dans sa vérité humaine et non plus dans son statut d'exemple institué et 'non négociable', si l'on peut dire. Diderot précisément négocie avec ses contemporains, avec lui-même, avec l'ordre et l'absolutisme préexistant à toute expression dont la sienne, négociation qui rend indispensable le recours à un genre un peu oublié à l'époque : le dialogue. En ce sens, s'il y a bien recours à nombre de topoi, la narrativité se dédouble en un récit manifeste et assez simpliste, à partir des diverses anecdotes de Jean-François Rameau, et un récit latent qui n'est autre que la biographie de ce neveu reconstruite par chaque lecteur dans son imaginaire propre.

La question de l'acquisition des savoirs est traitée de façon assez paradoxale : «Lui. — [D]ans ce pays-ci, est-ce qu'on est obligé de savoir ce qu'on montre ? Moi. — Pas plus que de savoir ce qu'on apprend. $»^{1}$ La relation pédagogique est, on le voit, entièrement à reconsidérer ; le lien entre la connaissance et sa transmission est rompu. A preuve : «Lui. - Vous donniez des leçons de mathématiques. Moi. — Sans en savoir un mot; n'est-ce pas là que vous en vouliez venir ? [...] J'apprenais en montrant aux autres, et $\mathrm{j}$ 'ai fait quelques bons écoliers $»^{2}$. L'expertise dans la discipline enseignée n'est pas indispensable pour former de bons esprits. Le neveu considère même que savoir nuit à enseigner, et que désapprendre est fondamental

${ }^{1}$ Diderot, Le Neveu de Rameau et autres dialogues philosophiques, p. 5.

${ }^{2}$ Ibid., p. 55. 
pour une initiation réussie. A propos de sa propre fille, le philosophe avoue qu'il ne lui apprend pas la musique, le chant ni la danse, mais « [à] raisonner juste, [...] de la grammaire, de la fable, de l'histoire, de la géographie, un peu de dessin et beaucoup de morale. [...] C'est sa mère qui se mêle de son éducation. $»^{3}$

Le neveu va plus loin que son interlocuteur, le philosophe : il faut étudier, s'exercer pendant des décennies avant d'être en mesure de transmettre un savoir (« Ce n'est qu'après trente à quarante ans d'exercice que mon oncle a entrevu les premières lueurs de la théorie musicale. [... Q]uand on ne sait pas tout, on ne sait rien de bien. ${ }^{4}$ Prenant l'exemple de la Physique («pauvre science ») le neveu pousse le pessimisme jusqu'à lui préférer l'ignorance, tant il reste de choses à comprendre. Manifestement, le questionnement ne concerne pas le rapport au disciple sensiblement le même en chaque personnage — mais le rapport du maître au savoir.

Le maître de musique introduit une donnée absente jusque-là - en tout cas chez Fénelon : la rétribution ( «L’heure se passait. Mon écolière me présentait le petit cachet, avec la grâce du bras et la révérence qu'elle avait apprise du maître à danser [...] et voilà ce qu'on appelait alors une leçon. $»^{5}$. L'ironie, voire la cruauté, est ici de faire évaluer par le musicien la leçon bien apprise du maître à danser. Mais à la page suivante, les années passant, la rétribution a pris une autre forme :

\footnotetext{
Lui. - Je donne ma leçon et je la donne bien : voilà la règle générale. Je fais croire que j'en ai plus à donner que la journée n'a d'heures, voilà l'idiotisme. [...] Autrefois je volais l'argent de mon écolier, oui, je le volais, cela est sûr. Aujourd'hui je le gagne, du moins comme les autres.

Moi. - Et le voliez-vous sans remords ?

Lui. - Oh! sans remords ! [...] Les parents regorgeaient d'une fortune acquise Dieu sait comment ; [...] Je les aidais à restituer ${ }^{6}$.
}

Ainsi est-on passé du vol au salaire, de la rétribution à la restitution, d'aucuns diraient de la justice au cynisme. Et pour cette donnée sociologique qui vient dénaturer la relation pédagogique, le neveu use d'un terme, qui ne lui vient d'ailleurs pas spontanément, l'«idiotisme moral ». C'est un peu comme s'il voulait masquer sa dépendance à l'égard du Capital sous une évolution commune à l'ensemble de ce

\footnotetext{
${ }^{3}$ Ibid.

${ }^{4}$ Ibid., p. 58.

5 Ibid., p. 60.

${ }^{6}$ Ibid., p. 63.
} 
qu'il appelle « les métiers ». L’irruption du domaine privé (la différence de condition entre le précepteur et « l'écolier ») dans la relation de maître à disciple se fait au fur et à mesure de la croissance des réalités économiques au sein du système social; pour être exact, il faudrait parler de croissance en visibilité 7 . Mais si l'on observe une démarcation grandissante entre le savoir et le contenu d'enseignement, entre l'état privé et le domaine public vécu comme pression économique, et surtout entre l'autonomie et la dépendance du maître, il reste que ce dernier demeure le maître et que le disciple ne change pas de rôle. La question de la liberté, et a fortiori celle d'un rôle éventuel de l'éducation dans l'émancipation des hommes n'est pas encore mûre, d'autant moins que l'éducation, en matière morale en tout cas, paraît même inutile : « [S]i la molécule voulait qu[e mon fils] fût un vaurien [...], les peines que j'aurais prises pour en faire un homme honnête lui seraient très nuisibles $[\ldots] »^{8}$. Le mentorat s'est effacé, l'étude semble prendre le pas sur l'éducation, et le savoir et la formation sont nettement découplés. On sent que la notion de transmission va devenir essentielle pour l'initiateur de l'encyclopédie, ainsi que l'adoption d'un principe de réalité touchant l'économie de l'enseignement.

\section{Jacques le fataliste (1765-1796) : le discours du maître}

Dans Jacques le Fataliste, "il existe un discours du maître », pour reprendre l'expression célèbre de Jacques Lacan'. Du maître, certes, mais en quel sens ? L'interlocuteur anonyme de Jacques est explicitement celui qui détient tout pouvoir sur son valet; et il l'est nominalement puisque ce terme de « maître » est le seul par lequel il est désigné. Exerce-t-il ce pouvoir ? L'œuvre ne donne pas à le penser. Le même Lacan disait du maître qu'il «a toujours raison parce qu'il est l'autre nom du Monde ${ }^{10}$. Qui est donc celui qui a toujours raison ?

\footnotetext{
7 Telle que la rêve le neveu : « Lui. — Et cet homme, qu'est-il devenu ? Moi. — S'il eût été sage, il eût fait fortune, la seule chose que vous ayez en vue. Lui. - Sans doute. De l'or, de l'or. L'or est tout, et le reste, sans or n'est rien. » (ibid., p. 114)

${ }^{8}$ « Si [mon fils] est destiné à devenir un homme de bien, je n’y nuirai pas. Mais si la molécule voulait qu'il fût un vaurien comme son père, les peines que j'aurais prises pour en faire un homme honnête lui seraient très nuisibles [...] » (ibid., p. 112).

${ }^{9}$ Jacques Lacan, Du discours psychanalytique, Conférence à l'université de Milan, 1972, dans Lacan in Italia-Lacan en Italie, 1978, p. 47.

${ }^{10}$ Remarque citée par Bernard Henri Lévy dans la présentation de son ouvrage La Barbarie à visage humain : voir http://www.bernard-henri-levy.com/jacques-lacan-2-33028.html
}

https://journals.uvic.ca/index.php/sator/index 
Dans une autre acception du mot, il y a du maître dans une relation à un disciple : il y a de l'instruction, un corpus à transmettre. Si Le Neveu de Rameau met pour la première fois en lumière les apories de la transmission, Jacques Le Fataliste va mettre celle-ci en situation, par un enchaînement de cas illustrant tantôt le désir, tantôt la vanité, de transmettre. A priori, le protagoniste va instruire autrui de son fatalisme (Jacques), tandis que le Maître fera connaître ce qui l'en distingue, sa croyance en un dieu, ou son adhésion au mouvement des lumières, peut-être. La vraisemblance plaide pour un maître omniscient dont le valet aurait tout à apprendre — dans le droit fil d'un Don Juan, tuteur de Sganarelle en rationalisme exacerbé. La réalité est peut-être tout autre.

\section{Jacques ou l'ignorance. Petit inventaire de situations pédagogiques}

Dès maintenant, il est à remarquer que ni Jacques ni son maître ne savent où ils vont ; on serait tenté de dire qu'ils ne connaissent ni leur destination ni leur destinée. Voilà d'ailleurs un point sur lequel le narrateur lui-même est bien peu informé : «Mais, pour Dieu, lecteur [...] est-ce que l'on sait où l'on va ? Et vous où allez-vous ? ${ }^{11}$. Quant à savoir ce que chacun des personnages veut apprendre, le lecteur est plutôt perplexe ; à coup sûr, ce qui lui vient à l'esprit, c'est que le Maître est très désireux de connaître ... l'histoire des amours de son valet. Et celui-ci, que regrette-t-il d'ignorer? La réponse ne se donne pas en termes de contenu, car c'est autre chose qu'il aspire à connaître, comme en témoigne ce passage :

\footnotetext{
Mon maître, on ne sait de quoi se réjouir ni de quoi s'affliger dans la vie. Le bien amène le mal, le mal amène le bien. Nous marchons dans la nuit au-dessous de ce qui est écrit là-haut, également insensés dans nos souhaits, dans notre joie, et dans notre affliction. Quand je pleure, je trouve souvent que je suis un sot. [... J'ai essayé] de me moquer de tout. Ah! si j'avais pu y réussir [cela m'aurait servi à] me délivrer de souci, à n'avoir plus besoin de rien, à me rendre parfaitement maitre de moi [...] J'y ai renoncé, j'ai pris le parti d'être comme je suis ${ }^{12}$.
}

Cette volonté d'être autre, autre que ce que je suis («Puis-je n'être pas moi ? Et étant moi, puis-je faire autrement que moi ? Puis-je être moi en un autre ? ${ }^{13}$, découle manifestement d'un non-savoir, et lorsque le maître l'oriente vers une autre volonté

\footnotetext{
${ }^{11}$ Jacques le Fataliste, p. 70.

${ }^{12}$ Ibid., p. 106-107. Je souligne.

${ }^{13}$ Ibid., p. 31.
} 
possible, devant laquelle la « résignation [est] plus facile et plus commode », Jacques ne relève pas, parce qu'il ne comprend pas.

Jacques ne prétend pas tout savoir, mais ne détaille que rarement ce qu'il ignore ${ }^{14}$. Cependant, au fil de l'œuvre, l'auteur glisse un certain nombre de 'situations pédagogiques' qui tournent en dérision une relation maître-disciple devenue désuète :

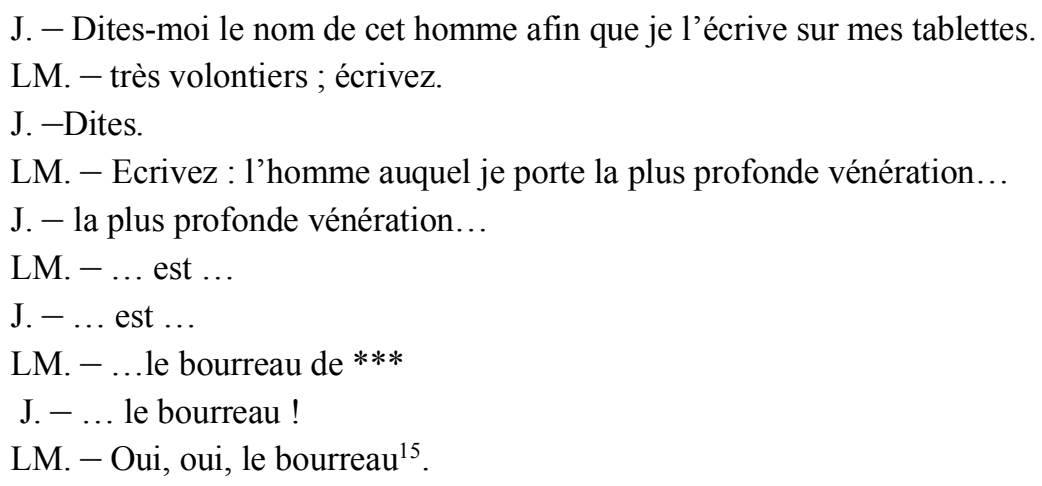

A cela s'ajoutent le moment de la colère pédagogique ( « LM. - ... et l'origine de la querelle ... ? J. - Ma foi, je l'ignore. LM. - Tu ignores le fond d'une affaire et tu t'en mêles ! », le moment du contrôle des acquis (« Pourrais-tu me dire ce que c'est qu'un fou, ce que c'est qu'un sage? [...] Et qu'est ce qu'un homme heureux ou malheureux ? [...] Et qui est-ce qui a écrit là-haut le bonheur ou le malheur ? », puis le moment où l'on ouvre un nouveau chapitre («Je vais t'apprendre à parler » et même la demande d'explication de l'élève (« J. - Vous êtes quelquefois si profond et si sublime que je ne vous entends pas. Ne pourriez-vous pas m'éclaircir cela par un exemple ? $»^{16}$. Certains commentateurs sont même allés plus loin en alléguant un pédagogisme généralisé de la part de l'auteur :

«Éducateur du genre humain », le philosophe-romancier ne veut ni ne peut se dispenser d'une certaine violence pédagogique que l'on retrouvera chez Brecht: il faut arracher le consommateur de fiction à une fascination engluante, le contraindre [...] à assumer le sens de

\footnotetext{
${ }^{14}$ Par exemple on a ceci : « Il y a là je ne sais combien d'os, de tendons, et bien d'autres choses qu'ils appellent je ne sais comment ...» (p. 27) Et pourtant, «[d]e tous [1]es lexiques, le plus employé est celui de la pratique médicale dont Diderot n'a cessé de suivre l'évolution [...] il accoutume à appeler toutes choses par leurs noms et, par sa vigueur univoque, tranche avec la duplicité du discours dévot. » : Éric Walter, Jacques le Fataliste de Diderot, 1975, p. 59. On remarque que plus loin : « Mon maître, il est écrit là-haut que [...] » est reformulé, humanisé, réinséré dans le monde d'en bas par «... que je l'écrive sur mes tablettes » qui prend des allures d'émancipation par l'apprentissage.

${ }^{15}$ Jacques le Fataliste, p. 95.

${ }^{16}$ Ibid., p. 112, 35, 309, 98.
}

https://journals.uvic.ca/index.php/sator/index 
ce qu'il lit. Truqué, caricatural, ce dialogue auteur-lecteur a donc pour but de démasquer la mauvaise foi du pacte romanesque ${ }^{17}$.

S'il est difficile de dire ce que Jacques voudrait apprendre, il n'est guère plus aisé de déterminer en quoi le maître est instruit. Quel est son domaine d'expertise ? On est presque étonné lorsqu'il fait valoir aux dépens de Jacques sa connaissance des chevaux : «J. - J'ai servi dans l'infanterie, et je ne m'y connais pas. LM. - Moi, j'ai commandé dans la cavalerie, et je m'y connais. $\rangle^{18}$. Là résiderait en partie l'originalité de Jacques le Fataliste: le Maître ne prétend pas tout savoir, lui non plus ; mais en tout, il se place par rapport au savoir : ses énoncés sont des modalités épistémiques. Face aux propos de Jacques qui sont majoritairement factuels, le Maître énonce rarement sans s'être préalablement positionné par rapport au savoir : «je crois que .../ tu (ne) me parais (pas) $\ldots /$ il faut que $\ldots /$ je pense que .../ tâche de $\ldots /$ je (ne) doute (pas) que .../ jugez-vous que .../ je présume que .../ je voudrais savoir ... / j'ai peur que ... / il me semble que ... / je gage que ... etc. ». Les deux personnages sont les produits de deux cultures, celle du fait et celle du degré de certitude ; la première a pour corollaire la foi inébranlable, la seconde un penchant pour une forme d'indécision qui n'est jamais éloignée de l'inaction: l'autorité a changé de camp.

\section{Bilan. Une relation maître - disciple inversée}

Le bilan de la situation se présenterait comme un cumul de plusieurs éléments inattendus : un maître sans nom, un disciple qui se fait formateur, un corpus à transmettre sous l'étiquette de fatalisme: je m'appelle Jacques, je serai votre mentor. Car Jacques est certainement plus proche d'un mentor au sens où il privilégie le savoir acquis par l'expérience, dont tout l'objectif est de faire remarquer que le Maître est dénué. Car ce dernier n'a vécu ni la guerre, ni la blessure physique, ni la victimisation voire l'humiliation auxquelles le valet a dû s'adapter autant que faire se

\footnotetext{
${ }^{17}$ É. Walter, Jacques le Fataliste de Diderot, op.cit., p. 31. D'autres commentateurs sont allés jusqu'à considérer les thèmes du cheval, voire du chien, comme symbolisation du dressage et de l'instruction. La référence à Brecht est intéressante, en ce sens que ce dernier, particulièrement dans Arturo Ui, invente la distanciation (Verfremdungseffekt) pour rompre le lien émotionnel entre l'acteur et le public - pacte tacite de croyance en ce que montre le spectacle, pacte par lequel le public se voit déposséder de sa liberté, et finit par adhérer aux codes symboliques de l'art bourgeois.

${ }^{18}$ Jacques le Fataliste, p. 62. Littré confirme que dans l'usage ancien, on appelle « maître » un soldat cavalier.
} 
peut. C'est dans ce contexte que se pose la question de la fameuse redistribution des rôles. En effet, la relation maître-disciple s'inverse au cours du récit, au point que cette évolution est l'un des traits distinctifs de l'œuvre : « Je crois mon maître que vous vous trompez », ou encore :

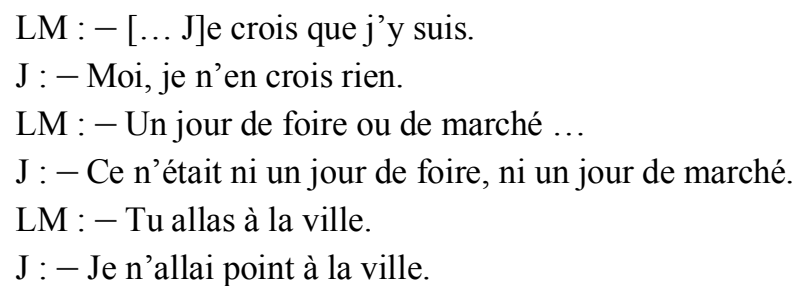

LM : - Et il était écrit là-haut que tu rencontrerais dans une taverne quelqu'une de tes créatures obligeantes; que tu t'enivrerais ...

$\mathrm{J}$ : - J'étais à jeun; et ce qui était écrit là-haut, c'est qu'à l'heure qu'il est vous vous épuiseriez en fausses conjectures; et que vous gagneriez un défaut dont vous m'avez corrigé, la fureur de deviner, et toujours de travers ${ }^{19}$.

Manifestement, dans ce passage, les rôles ont été inversés : celui qui est dans l'erreur, qui a besoin d'être instruit, ce n'est plus le valet. Certes, en reprenant à son compte le leitmotiv de Jacques («il était écrit là-haut»), le Maître met en danger le socle rationaliste qui jusque-là lui a conféré sa position dominante ; mais Jacques lui montre avec beaucoup d'à propos que l'élève a su dépasser ... le maître en rejetant les « fausses conjectures » et « ce défaut dont [celui-ci l'a] corrigé, la fureur de deviner. » C'est un argument fondamental car on y trouve une rare allusion à la possibilité de transformer l'homme par l'éducation, ce qui, jusque-là (dans Le Neveu de Rameau, par exemple) était sérieusement mis en doute. En outre, s'il est vrai qu'il s'agit bien d'enseignement, c'est moins en raison d'un contenu que des procédures pour y parvenir : prééminence du fait sur l'interprétation, foi en l'expérience réelle plutôt qu'en ses possibles ${ }^{20}$.

Des deux personnes en dialogue, il semble bien que le Maître, paradoxalement, soit le moins éducable. Il en convient lui-même : la peinture, « je n'y entends rien du tout »; ou dans cet échange :

$$
\begin{aligned}
& \mathrm{J}:-[\mathrm{V}] \text { ous signâtes les lettres de change ? } \\
& \mathrm{M}:- \text { Il est vrai. }
\end{aligned}
$$

\footnotetext{
19 Jacques le Fataliste, p. 101, 225-226. Reformulé plus loin : « Mon maître, il est écrit là-haut que $[\ldots]$ tant que vous vivrez vous [...] devinerez de travers. » (p. 227).

${ }^{20}$ Cet argumentaire n'est pas sans analogie avec le raisonnement que suit Kant, lecteur de Hume, sur l'existence de Dieu. Cf. Critique de la raison pure, Dialectique transcendantale, ch. III, 4e section.
} 
J. :- C'est l'usage des pères, lorsque leurs enfants partent pour la capitale de leur faire un petit sermon. Ne fréquentez point mauvaise compagnie ; [...] et surtout ne signez jamais de lettres de change.

$\mathrm{M}:-$ Que veux-tu, je fis comme les autres ; la première chose que j'oubliai, ce fut la leçon de mon père ${ }^{21}$.

Comme les autres, comme tout le monde : en raison de quel privilège le maitre seraitil omis par le «grand branle du monde »? L'écriture abolit les privilèges (n'est-ce pas l'écriture qui, en assurant à Jacques l'égalité, lui a donné sa liberté : «il me semble que [...] tu parles plus librement $» ?)^{22}$; le maître, quant à lui, est devenu disciple.

Et chacun de comprendre que c'est plutôt le maître qui souhaite s'instruire; l'insistance avec laquelle il ramène toujours Jacques au « récit de ses amours » atteste de son désir d'apprendre -même si pour lui il s'agit à peine d'apprentissage, sûrement d'acquisition de connaissance; en cela il se distingue de l'expérientialisme de Mentor. On aura d'ailleurs remarqué que lorsque Jacques signale au Maître ses erreurs, c'est à chaque fois sur le thème des amours : «[Mon maître, ...] vous vous trompez. $»^{23}$. La réponse est révélatrice du domaine sur lequel il veut être éclairé : « $[\mathrm{T}] \mathrm{u}$ crois que je passerai trois mois dans la maison du docteur avant que d'avoir entendu le premier mot de tes amours? $»^{24}$.

L'inversion de la relation maître-disciple atteint son paroxysme lorsque Diderot poignarde son personnage sur la question, précisément, de l'éducation. On a généralement assez peu commenté ce que le Maître fait du fils que lui offre le destin, en l'occurrence la rouerie de « son intime ami le chevalier de Saint-Ouin ». Le texte est sans équivoque :

On me condamna $[\ldots]$ à pourvoir à $[\ldots]$ l'éducation d'un enfant provenu des faits et gestes de mon ami [...] Il aura bientôt dix ans. Je l'ai laissé tout ce temps à la campagne, où le maître d'école lui a appris à lire, à écrire et à compter. [... J]e profite de la circonstance pour payer à ces gens ce qui leur est dû, le retirer, et le mettre en métier ${ }^{25}$.

\footnotetext{
${ }^{21}$ Jacques le Fataliste, p. 218, 260.

22 Ibid., p. 264 ; Jacques s'enhardit jusqu'à dire au maître son fait : « Monsieur, cela est bien fort. Vous avez donc été brave une fois dans votre vie? » (p. 268).

${ }^{23}$ Ibid., p. 101.

${ }^{24}$ Ibid., p. 102.

${ }^{25}$ Ibid., p. 302 ; C'est dans Jacques le Fataliste la seule allusion à l'économie de l'enseignement, car la question du savoir ne se pose plus dans les mêmes termes que dans Le Neveu; à rapprocher de cette autre remarque de Lacan : «Ce qui s'opère du discours du maître antique à celui du maître moderne qu'on appelle capitaliste, c'est quelque chose qui s'est modifié dans la place du savoir » (Jacques Lacan, « Leçon du 17 XII 1969 », L'envers de la psychanalyse, 1991, p. 34)
}

https://journals.uvic.ca/index.php/sator/index 
Qui pourrait, dans ces conditions, garder le titre de maître ? Condamné à éduquer un enfant — la formule est à l'opposé des convictions de l'auteur lui-même - il confie, contre monnaie sonnante, cette charge à un maître au sens propre (un «maitre d'école ») pour finalement laisser ce jeune humain entre les mains d'un patron ${ }^{26}$. À ce moment, la destination des deux personnages devient claire pour tout lecteur: rechercher l'enfant du destin et le soustraire à davantage d'instruction; chacun dès lors est à même de reconnaître qui est déchu de son titre de maître (ni guide, ni mentor, ni formateur), et d'identifier Jacques, celui qui assume son humanité malheureuse, à une figure salvatrice («c'est lui qui nous a sauvé la vie à tous »)²7.

\section{Désir d'apprendre ou désir de transmettre?}

Que transmet Jacques ? De quel savoir le Maître est-il en quête ? Sur son vœu d'apprendre, il y a trois lectures possibles : ce désir peut provenir d'une curiosité vaguement érotisée, ou d'un intérêt pour la vie du 'petit peuple', ou d'une recherche de validation de l'une des thèses en présence touchant la liberté humaine.

Jacques se permet de dire clairement au maître que sa curiosité n'est d'aucune utilité :

Et voilà le commencement, le milieu et la fin de la perte de mon pucelage. À présent, Monsieur, je voudrais bien que vous m'apprissiez le but moral de cette impertinente histoire.

LM. - À mieux connaître les femmes.

J. - Et vous aviez besoin de cette leçon?

LM. - À mieux connaître les amis ? [...] À mieux connaître les pères et les enfants. [...] Quel que soit le récit que tu m'as promis après celui-ci, sois sûr qu'il ne sera vide d'instruction que pour un sot $[.]^{28}$.

«Apprendre, leçon, connaître, instruction », on jurerait que le questionnement sur l'éducation est ici essentiel. Or, à cet égard, la situation est ici plus complexe puisque l'instructeur ne trouve aucun intérêt à ce qu'il transmet. Quant à celui qui se réjouit d'apprendre, il est d'ordinaire le dépositaire des savoirs sur lequel tous s'accordent. Qu'en est-il donc de ce désir d'apprendre du maître? Est-ce simple curiosité, y compris sous une forme érotisée -un voyeurisme déguisé, en quelque

\footnotetext{
${ }^{26}$ On est loin de l'éducation donnée par sa mère à la fille du philosophe : raisonnement, grammaire, histoire, dessin etc. (Le Neveu de Rameau, p. 55).

${ }^{27}$ Jacques le Fataliste, p. 316.

${ }^{28}$ Ibid., p. 235-236.
}

https://journals.uvic.ca/index.php/sator/index 
sorte ? À en croire la psychanalyse, le désir de connaître est sexuel, au sens freudien du terme.

Une seconde hypothèse serait une explication sociologique de ce désir d'apprendre ; elle serait due à un intérêt, caractéristique du second tiers du XVIII ${ }^{\mathrm{e}}$ siècle français, pour le vécu des autres, et particulièrement des hommes alors qualifiés 'de petite condition'. C'est un temps où l'on découvre les plus pauvres, à l'instar du Comte Almaviva qui s'interroge sur les racines de la «philosophie » de Figaro : «Qui t'a donné une philosophie aussi gaie ? [Fig.] L'habitude du malheur. Je me presse de rire de tout, de peur d'être obligé d'en pleurer. Que regardez-vous donc toujours de ce côté ? $»^{29}$. C'est un temps où au sein même du Tiers-État, on commence à se considérer avec intérêt et on se cherche des points communs plus que des différences. Diderot lui-même, dans Le Neveu, demandait comment l'on cesse d'être pauvre : « Il y eut un temps où vous n'étiez pas cossu comme aujourd'hui. Moi. - Je ne le suis encore trop. Lui. - Mais vous n'iriez plus [... e]n redingote [...] éreintée par un des côtés $[. .$.$] les bas de laine noirs recousus par-derrière avec du fil blanc. »^{30}$.

Il se pourrait enfin que le Maître soit à la recherche de la confirmation éventuelle d'une thèse sur les déterminants de l'action, voire de l'humaine condition, et particulièrement la validation ou non d'une emprise transcendante (providence, destin, divinité ?...). Jacques a-t-il raison d'être fataliste ? Et la probabilité qu'il ait tort nous conduit-elle à supposer une autre instance? Au plan formel, les conséquences de chaque hypothèse serait, pour la première, le récit libertin qui, malgré de fréquentes interruptions, se poursuit d'un bout à l'autre de Jacques le Fataliste; et pour la seconde, une forme romanesque, dans le ton d'un picaresque tardif (Cf. Le Sage : Gil Blas). La dernière hypothèse serait intimement liée à une forme oubliée - à quelques exceptions près - mais parfaitement adéquate pour 'publier' une quête de sens : le dialogue.

Jacques le Fataliste dans la lignée des dialogues post-socratiques. Que Platon n'ait pas été lu dans sa langue n'est pas un fait nouveau: au milieu du $\mathrm{XVI}^{\mathrm{e}}$ siècle, on commence tout juste à le traduire en latin, rarement dans les langues vernaculaires et il ne s'agit aucunement des œuvres complètes. Anne-H. Klinger-Dollé signale qu'il s'agit essentiellement des dialogues sur l'immortalité de l'âme et de ceux sur

\footnotetext{
${ }^{29}$ Beaumarchais, Pierre-Augustin Caron de, Le Barbier de Séville (1772-1775), 1982, I, 1, p. 72-73.

${ }^{30}$ Le Neveu de Rameau, p. 55.
}

https://journals.uvic.ca/index.php/sator/index 
l'amour ${ }^{31}$. Il faut attendre le XVIII ${ }^{\mathrm{e}}$ siècle tardif pour que s'accroissent la lecture dans le texte et l'habitude de traduire directement en français (Diderot était au nombre de ces précurseurs). En revanche, l'intérêt du dialogue platonicien avait déjà ses défenseurs. L'article «Platonisme» de l'Encyclopédie met en équation le style du philosophe grec et cette notion de «goût» qui, de Shaftesbury (1711) à Baumgarten (Aesthetica, 1750), aura été la valeur esthétique primordiale : «celui qui n’est pas sensible au charme [des] dialogues [de Platon] n'a point de goût. $»^{32}$ Opter pour la forme dialoguée c'est, de la part de Diderot, un acte de confiance en cette faculté perçue comme naturelle, subjective, de juger du beau.

Mais le dialogue est aussi un mode de connaissance; en tant que méthode d'investigation intellectuelle, échappant aux contraintes de structure propres aux autres formes littéraires, il sert, non à informer, mais à former. C'est tout l'enjeu de la dialectique propre à Jacques le Fataliste. Il est à remarquer qu'à la moindre tentative de raisonnement, l'auteur, se limite au cadre défini par la méthode dite élenctique celle qui assimile la vérité à la non-contradiction, c'est d'ailleurs en ce sens que Jacques est présenté comme «philosophe $»^{33}$. Or l'elenchus (๕̌ $\left.\lambda \varepsilon \gamma \chi \circ \varsigma\right)$ post-socratique à l'œuvre dans Jacques le Fataliste suscite en chaque interlocuteur, qui, bien sûr, est aux aguets de la moindre contradiction pour ne rien dire des moments où il la provoque, davantage de curiosité intellectuelle, ouvrant ainsi la voie à la recherche d'une vraie sagesse. Chacun veut, malgré soi, comprendre les idées de l'adversaire. C'est pourquoi le second aspect caractéristique du dialogue est sa nature heuristique : il n'y a pas de vérité préalable. Car même si Jacques pose en principe le déterminisme absolu comme ordre du monde, il ne s'agit pas là d'une vérité ; le Maître s'en amuse, et Jacques admet que d'autres puissent en douter. Il y a donc deux croyances qui s'évaluent mutuellement, mais on ne saurait parler de vérités ; elles sont peut-être en devenir, et c'est cela qui emporte l'adhésion du spectateur ou du lecteur, comme c'était le cas pour Le Neveu. Amor Cherni a remarquablement synthétisé cet aspect de l'œuvre :

\footnotetext{
${ }^{31}$ Anne-Hélène Klinger-Dollé « Platon latin, Platon françois : quelques traductions de la Renaissance, entre philosophie morale et fictions poétiques », Université de Toulouse-Le Mirail, équipe PLHERASME, http://plh.univ-tlse2.fr/servlet/com.univ.collaboratif.utils.LectureFichiergw?ID...ID... . ${ }^{32}$ Encyclopédie, art. « Platonisme », 1778, t. 26, p. 197 ou Encyclopédie, art. «Platonisme », 1751, t. 12, p. $746:$ https://fr.wikisource.org/wiki/L'Encyclopédie/1re_édition/PLATONISME

${ }^{33}$ En tant que méthode de réfutation, l'elenchus ne peut établir que les vérités relevant de la cohérence. Il suffit que les propositions avancées par l'interlocuteur soient cohérentes (au sens où elles ne se contredisent pas) pour que la thèse soit tenue pour vraie.
}

https://journals.uvic.ca/index.php/sator/index 
Ce qui intéresse Diderot dans le dialogue dit socratique, c'est [... cette tentative de mettre aux prises] deux pensées dont aucune n'est soumise à une vérité déjà acquise et imposée comme préalable au dialogue [...], un double décentrement qui porte chacun des partenaires hors de lui-même et qui arrache le discours à la pure subjectivité, $[\ldots]$ une alternance ininterrompue entre le dedans et le dehors, le particulier et le général, l'individuel et le collectif $f^{34}$.

La dialectique à l'œuvre dans Jacques le Fataliste est-elle celle de Platon ? N'a-t-on pas évoqué d'autres inspirateurs : Lucien de Samosate, Fontenelle, Voltaire? Plus généralement, ce n'est pas le moindre des paradoxes de Diderot que de recommander l'étude de Platon autant qu'il la déconseille : « Il y a plus à profiter pour un homme de génie dans une page de cet auteur que dans mille volumes de critiques. $»^{35}$. La restriction que comporte la formule «homme de génie» est ici plus explicite encore : « les ouvrages de Platon, abrégés de science universelle, sont trop profonds, même pour les maîtres, et je les crois aussi propres à gâter l'esprit qu'à perfectionner le style. ${ }^{36}$ L'étude de Platon est clairement préconisée pour une élite intellectuelle, les esprits en cours de formation devant s'arrêter à des aspects soigneusement bornés, parmi lesquels on n'est pas surpris de retrouver le travail du style. Des raisons esthétiques feraient de Platon le Mentor des hommes ordinaires mais une fraternité de génies qui ferait de lui le Mentor de tout penseur. Philosophe pour les uns, 'bon homme' pour les autres, Jacques est-il de ceux-là ? Il est à remarquer qu'il «se réclame du spinozisme sans avoir lu Spinoza » et que, sans le Maître, Jacques n'aurait jamais entendu parler de Socrate ${ }^{37}$. Une fois que la version abrégée du Phédon lui a été communiquée par son maître, Jacques assimile l'attitude de celui-ci à celle d'un « prophète », comme s'il le plaçait au rang des détenteurs du « grand rouleau ». On le voit donc à la fois apte à recevoir les plus hauts enseignements mais inapte à se défaire de ses habitudes de distorsion de toute idée nouvelle. Et d'ailleurs, cet épisode de philosophie antique n'aura plus aucune incidence sur les actions ou les pensées des interlocuteurs -la leçon aura été vaine. Télémaque au contraire retient les leçons de Mentor et cherche à les appliquer dès que l'occasion se présente.

On voit que le choix du dialogue et l'implicite platonicien qui le sous-tend ne sont pas étrangers à quelques pétitions de principe concernant l'éducation. Celle-ci doit

\footnotetext{
${ }^{34}$ Amor Cherni, Diderot. L'Ordre et le devenir, 2002, p. 26.

${ }^{35}$ Encyclopédie (article « Platonisme », éd. A.-C. Briasson, op. cit., 1751, t. 12, p. 746.

${ }^{36}$ D. Diderot, « Plan d'une université pour le gouvernement de Russie », dans CEuvres complètes, 1875, p. 491.

${ }^{37}$ É. Walter Jacques le Fataliste de Diderot, op.cit., p. 70 ; Jacques le Fataliste, p. 97-98.
} 
concerner tous les enfants quels qu'ils soient, y compris ces pauvres dont J.-J. Rousseau affirmait qu'ils en étaient dispensés ${ }^{38}$; cependant, il convient de ménager des degrés, le plus élevé (« l'état de savant») ne devant s'adresser qu'à une minorité ; il convient également de tenir compte, cette fois en accord avec Rousseau, du fait que la quantité de savoir ne conduit pas nécessairement à la vertu - loin s'en faut. En outre, il reste entre le peuple et tout philosophe un contentieux, une dette non soldée, dont la mort de Socrate n'est que l'aspect le plus tragique (« [1]e philosophe, dit Le Maître, [est] une race d'homme odieuse aux grands [...], aux peuples, de tout temps les esclaves des tyrans qui les oppriment. ») $)^{39}$. Par la voix du narrateur, Diderot se cache à peine de s'identifier à Socrate, de même que, dans l'article cité, deux allusions le rapprochaient singulièrement de Platon : si dans « homme de génie » on pouvait en effet pressentir les contours d'un autoportrait de Diderot, il semble bien que dans «l'abrégé de science universelle» on trouverait une délinéation du projet [en]cyclopédique commun à Chambers et à l'enfant prodige de Langres.

Il y a donc manifestement un lien fonctionnel indissoluble entre le mentorat, le dialogue, et la figure de Platon; on ira jusqu'à dire que la structure dialoguée, et particulièrement cette propension «élenctique» caractéristique des premiers dialogues de Platon, était la condition nécessaire et suffisante pour rendre faisable et crédible l'inversion des rôles de maître et disciple. Il reste maintenant à comprendre les raisons qui motivèrent cette inversion.

\section{La question prométhéenne}

Sans quitter l'univers platonicien, je propose d'étudier la reprise, dans Jacques le Fataliste, d'une question qui est fondamentale chez Socrate : la possibilité ou non d'enseigner la vertu. Car, on l'oublie souvent, c'est cette incertitude qui conduit Platon à évoquer la figure de Prométhée ${ }^{40}$. Or c'est aussi la dernière grande transcription du mythe avant celles du monde hellénistique puis romain. Usant d'un procédé dont s'est souvenu Diderot, Protagoras répond à une question par un récit, qui commence par le moment où les dieux façonnèrent les espèces ; Epiméthée avait

\footnotetext{
${ }^{38}$ Il y a là un point commun avec Le Maître qui, on l'a vu, soustrait son fils non désiré à davantage d'instruction « pour le mettre en métier » (ibid., p. 302).

${ }^{39}$ Ibid., p. 96-97.

40 Platon, Protagoras, 1923, 320b-322d, p. 34-37.
} 
choisi de finir par la race humaine mais ne put la doter, ayant épuisé la totalité des facultés qu'il avait pour tâche de distribuer espèce par espèce. Prométhée eut alors l'idée de voler à Héphaïstos et Athéna le feu et l'art au profit des hommes qui depuis se savent «en affinité avec les dieux ». Mais il ne put voler à Zeus la science politique. Celui-ci, plus tard, leur fit porter par Hermès, dans le seul but d'éviter l'extinction de l'espèce, la justice et la pudeur. C'est pourquoi, conclut Protagoras, la vertu peut s'enseigner. En somme, les dieux avaient écrit sur « un grand rouleau » la capacité à transmettre de génération en génération le savoir moral.

A l'origine, Hésiode avait mêlé les mêmes personnages à un récit assez différent. Puis vint Eschyle, qui corrigea la vision hésiodique ; et si l'on ne peut assurer que Diderot connaissait le Protagoras de Platon, on sait de manière certaine qu'il avait lu le grand tragédien grec. Contre Fontenelle, lequel voyait en Eschyle « une manière de fou qui avait l'imagination très vive et pas trop réglée $\|^{41}$, Diderot louait le poète « épique et gigantesque », ajoutant qu'il se refusait "à mettre dans l'Encyclopédie un écrit où Eschille étoit traité de fou $»^{42}$.

On ne pourra faire l'impasse sur le rapprochement avec Jacques le Fataliste (17651796), qui du point de vue que j'ai choisi est un peu l'inverse du Neveu, mais cet exercice a déjà été fait de manière si concluante qu'il paraît plus fructueux d'envisager un autre repère. Le Théâtre des Grecs du père Brumoy paraît en $1747^{43}$; Diderot y découvre Eschyle, malheureusement sous forme de résumés incluant quelques vers traduits, et particulièrement le Prométhée, qu'il commente et dont il diffuse les richesses dans son Encyclopédie. Il approuve l'auteur de l'article (Louis de Jaucourt) qui désigne le fils de Japet comme « le premier qui enseigna aux hommes », « qui avait formé l'homme », « il est l'inventeur de tous les arts, l'auteur de tout ce qu'il y a de connaissances utiles dans le monde, et cependant il n'a pas le pouvoir de se délivrer de la tyrannie de Jupiter $»^{44}$. Le lien est fait entre le premier des Guides,

${ }^{41}$ Remarques sur quelques comédies d'Aristophane, sur le théâtre grec, dans Fontenelle, Euvres, Paris, 1766, t. IX, p. 447.

${ }^{42}$ Cité par Abbé Trublet, Mémoires pour servir à l'histoire de la vie et des ouvrages de MM. de Fontenelle et de La Motte, tirés du Mercure de France, 1761, p. 172. Notre analyse suit celle de Raymond Trousson, Le thème de Prométhée dans la littérature européenne, 2001.

${ }^{43}$ Révérend Père Brumoy, Le Théâtre des Grecs, 1730, réédition en 1747 avec corrections et additions. Lire sur ce point : Malika Bastin-Hammou, «Introduction. Brumoy, pédagogue et passeur du théâtre grec », Anabases, 14, 2011, p. 27-41 et Claire Lechevalier, L'Invention d'une Origine. Traduire Eschyle en France de Lefranc de Pompignan à Mazon : Le Prométhée enchaîné, 2007.

${ }^{4}$ Article consultable sur le site de l'Encyclopédie (numérisée) de Diderot et d'Alembert, à l'adresse suivante : 
pour ainsi dire, et la destinée des hommes qui est de conduire le mouvement de l'Histoire dans le sens du Progrès, dont la première étape est de s'affranchir de toute tyrannie. Dans une représentation chronologique de cet aspect majeur de l'œuvre de Diderot, la première période serait :

1735-1740 Pandore (Voltaire écriture) $)^{45}$, avant 1747 (Père Brumoy),

1750 Rousseau contre Prométhée,

1753 Prométhée Houdar de La Motte,

1762 Pandore (opéra d'après Voltaire, représenté),

1762-1773 (Le Neveu de Rameau),

1 XII 1765 «Prométhée », article de Louis de Jaucourt in: Encyclopédie, et enfin

1765-1796 (Jacques le Fataliste).

Ce qui paraît être une inflexion de la figure du maître, et qui est incontestable (« Ce drôle-là fait de moi tout ce qu'il veut », dit le maître ${ }^{46}$ ), est peut-être dû à la lecture de Prométhée et à la prégnance accrue de ce qu'il signifie symboliquement pour qui veut se représenter l'évolution de l'humanité.

Il faut dire que le Prométhée de Diderot est assez unique en son temps; bien peu le comprennent comme un libérateur, encore moins comme un protecteur de l'humanité. On trouverait chez Voltaire les prémisses de la lecture qu'en fait Diderot. Mais la prééminence des arts et techniques, la conquête de l'énergie au service du maintien de l'espèce et son obligation de progrès, la familiarité avec le divin sans en concevoir de dette, ceci est associé à une symbolique prométhéenne qui n'apparaît chez aucun autre contemporain. Former l'homme - dans tous les sens de ce verbe - sans distinction de rang ou de pouvoir, sans non plus parvenir à l'affranchir de ce qu'il faudrait appeler « un sentiment du destin », c'est une tonalité nouvelle que l'on entend dans Jacques et qui sonne comme un écho du mythe prométhéen.

http://xn--encyclopdie-ibb.eu/index.php/science/1001261056-astronomie/921231911-PROMETHEE

45 Voltaire présente d'ailleurs son livret de Pandore, entrepris dès 1739, comme " un opéra philosophique qui devrait être joué devant Bayle et Diderot. » (Voltaire. Lettre à Laborde, 4 novembre 1765, D12966, édition de Théodore Besterman, Correspondence and Related Documents, 1968-1978, cité par Béatrice Ferrier, "Pandora, ou le bonheur originel selon Voltaire », Anabases, 19, 2014, p. 313-325.

${ }^{46}$ Jacques le Fataliste, p. 272.

https://journals.uvic.ca/index.php/sator/index 


\section{Le mentorat et la crise}

Si la référence à Prométhée a pris aujourd'hui un sens presque à l'opposé du mythe initial (il est devenu un homme, plus conscient que les autres et prêt à se sacrifier pour libérer ses frères !), le souvenir de Mentor a suivi un chemin inattendu. En témoigne cette page d'accueil d'un très sérieux site de ressources humaines :

Le mot mentor est utilisé comme nom commun pour désigner une personne qui partage son expérience, ses connaissances, ses compétences avec quelqu'un de plus novice. Le transfert d'expérience $[\ldots$ se pratique tous les jours ...] de façon informelle. [... Le] mentoring correspond à un programme formel, mis en place par une organisation dans le but de développer la carrière ou les connaissances d'une personne (mentoré). [...N] ous pouvons constater des différences [entre mentoring et coaching] Le coaching est orienté objectif. Le mentoring est quant à lui orienté relation [...] Le coaching vise le court terme. [...] Le mentorat vise toujours le long terme. [...] Le coaching est axé sur la performance. [...] Le mentoring est quant à lui axé sur le développement personnel ${ }^{47}$.

Pauvre Télémaque! Malgré les dérives que l'on pressent dans cette utilisation de la référence à Mentor, on n'en a manifestement pas terminé avec la quête d'une méthode parfaite pour transformer la personne plutôt qu'accroître son savoir.

Le 4 février 1962, Orville Prescott, critique littéraire au New York Times, signalait à ses lecteurs que Mentor, Télémaque et Prométhée n'étaient pas les seuls à se glisser dans notre vie quotidienne, réelle ou rêvée :

A la page 156 du nouveau roman de John Updike 'The Centaur', un ivrogne qui descend en titubant ou en vacillant une rue sombre d'Alton, petite ville de Pennsylvanie, insulte deux passants de façon particulièrement agressive. À la page 301, dans un "Index des allusions mythologiques », ce personnage sans importance est identifié au grec Dionysos. Ils sont également cinquante six autres dieux et figures mythologiques à être identifiés aux citoyens modernes de Pennsylvanie en 1957. C'est un jeu facile à jouer pour un romancier. Une femme qui n'a rien de plus que les autres est évidemment Vénus; il faut que tel mécanicien soit Vulcain. Le principal d'une école sur laquelle il fait régner son autorité n'est autre que Zeus ${ }^{48}$.

\footnotetext{
${ }^{47} \mathrm{http} / / /$ www.winchrh.fr/coaching-et-mentoring-des-similitudes-et-des-differences/

${ }^{48}$ Trad. M.-G. Gouverneur, d'après l'original : "On page 156 of John Updike's new novel, "The Centaur," a drunkard, weaving and tottering down a dark street in the Pennsylvania city of Alton, offensively insults two passers by. On page 301 in a "Mythological Index" this unimportant character is identified with the Greek god Dionysos. Some 56 other gods and mythological characters are also identified with modern citizens of Pennsylvania in the year 1947. It is an easy game for a novelist to play. A woman no better than she should be is obviously Venus. A mechanic must be Vulcan. A school principal wielding authority over others is Zeus. » Adresse de l'article original : http://www.nytimes.com/1962/02/04/books/updike-centaur.html
}

https://journals.uvic.ca/index.php/sator/index 
Ce roman atypique fut d'abord reçu, cet article en témoigne, comme un jeu inutile après Ulysses de J. Joyce ${ }^{49}$. Il obtint l'année suivante la plus haute distinction américaine pour une œuvre littéraire (traduite en français depuis 1965). A la seconde ligne du récit, on lit : «Éclat de rire général dans la classe. »; à la dernière ligne : «... bien peu de mortels [...] prennent les étoiles pour guides $»^{50}$. Cela suffit à démontrer que le roman est entièrement enserré, entenaillé, dans la relation pédagogique ; mais cette fois, il s'agit des rapports qui se tissent entre un professeur d'école publique, George Caldwell, dont l'épouse est aussi professeur, et son fils, également élève dans ce même établissement. Cependant, la relation est à ce point inversée que celui qui devrait être le maître (la critique contemporaine le désigne comme «role model», et son avatar mythologique est Chiron le précepteur) se découvre dans le regard de son fils (qui se veut son disciple) et se sauve ainsi de devoir considérer sa vie de professeur comme un échec. George est un homme bon, qui sent sa mort prochaine autant que son inadaptation à l'existence; de rares événements le transforment dont les quelques jours qu'il passe en tête à tête avec Peter, son fils, du fait d'une tempête de neige, la rencontre avec Véra (Vénus) qu'il séduit (ou qui le séduit), une conversation avec un auto-stoppeur qui se prend d'amitié pour ce chauffeur presque sans destination dont il met l'âme à nu et dont il fera connaître au monde la bonté. Les quelques actes qui prouveraient sa liberté sont aussitôt source de culpabilité : trop peu de volonté pour résister à Vera alors qu'il est marié, devenu professeur de science sur intervention d'un ami désireux de le sortir de sa dépression, détruit de l'intérieur par une image désastreuse de lui-même (au dentiste perplexe devant l'état de sa dentition [«Quel dommage ! l'émail de vos dents est moins fort que les racines »], Caldwell répond : «Telle est l'histoire de ma vie. Grand par les pieds, mais faible de la tête. $»^{51}$. Et surtout, la remise en question du bien fondé d'une relation triangulaire fructueuse entre l'homme, la nature et

\footnotetext{
${ }^{49} \mathrm{Du}$ fait des dimensions limitées d'un article, il a paru plus aisé de condenser The Centaur, par ailleurs centré sur la question de l'éducation et de la filiation. Mais une autre œuvre méritait au moins autant d'attention, il s'agit des épisodes de Ulysses que Joyce intitule «Scylla And Charybdis », et «Ithaca ». On y retrouve en effet la figure du maître d'école (Stephen Dedalus -le père d'Icare), un Platon transformé en mystique (AE / G.W. Russell), le personnage de Leopold Bloom, dont le nom évoque l'épanouissement qui suit la germination mais qui est hanté par la mort de son fils, la forme dialoguée reçue de Socrate etc. ; on voit donc que les thèmes de la $\pi \alpha \_\delta \varepsilon i ́ a$, de la transmission, du vécu individuel de la culture et des origines auraient justifié de consacrer plus à cette œuvre unique.

${ }^{50}$ "The class burst into laughter»; à la dernière ligne : "few mortals [...] sit as students to the stars ». Les citations sont extraites de The Centaur, Harmondsworth, Penguin books, 1963.

${ }^{51}$ "It's a shame, the caps of your teeth aren't as strong as your roots. - "That's the story of my life," Caldwell says. "Bigfeet, weak head". » : The Centaur, éd. cit., p. 225.
} 
l'éducation est aussi dissonante qu'archaïque, tant cet aspect de la culture américaine est perçu négativement : «Nos pères fondateurs dans leur grande sagesse décidèrent que les enfants représentaient pour leurs parents une contrainte contre nature. Ils dotèrent donc le pays de prisons qu'on appelle des écoles, équipées d'instruments de torture qu'on nomme l'éducation. $»^{52}$.

John Updike expliqua plus tard qu'il avait choisi la figure mythique de Chiron, le Centaure, comme étant le seul exemple de sacrifice de soi de la mythologie. Car de même que Chiron obtient de Zeus de devenir mortel afin de sauver Prométhée de ses souffrances - et Zeus lui rendra hommage en faisant de lui la constellation du Sagittaire d'où il aide à régler nos destinées -, de même George accepte la mort pour ne plus être un obstacle à l'épanouissement de son fils, Peter/Prométhée.

Ainsi la figure de Prométhée a-t-elle une nouvelle fois remis en cause l'homéostasie de la relation de maître à disciple. Tout comme on a vu ce héros transformer la conception "post-mentorienne » de l'éducation que l'on trouve chez Diderot, de même au milieu du $X^{\mathrm{e}}$ siècle, le désir d'enseigner pousse le maître à choisir ce qui est pour lui le degré ultime de la sagesse : la mort. Car, malgré les apparences, Updike s'inscrit dans le droit fil de la problématique instaurée par Diderot : la corrélation de l'éducation, de la liberté et du déterminisme. Quant à l'aspect politique inhérent à la figure du mentor, celui de Fénelon au premier chef, il s'est à première vue évanoui ; mais est-ce vraiment le cas ? Il est en effet significatif que pour la génération qui voit mourir sous ses yeux son plus jeune président (qui est aussi un jeune père) génération qui suit celle sacrifiée sur l'autel de la seconde guerre mondiale - , ce qui distingue la figure du père de celle du formateur, du guide, s'estompe graduellement : le premier devoir de chaque homme est désormais de transmettre, d'assurer la formation de ses héritiers, quitte à y laisser la vie.

Michel-Guy GOUVERNEUR, Docteur de l'Université d'Amiens, ancien chercheur associé au laboratoire CURAPP UMR 7319.

\section{BIBLIOGRAPHIE}

\footnotetext{
52 « The founding fathers in their wisdom decided that children were an unnatural strain on parents. So they provided jails called schools, equipped with torture called education. », Ibid., p. 125.
} 
BASTIN-HAMMOU, Malika, « Introduction. Brumoy, pédagogue et passeur du théâtre grec », Anabases, 14, 2011, p. 27-41.

BEAUMARCHAIS, Pierre-Augustin Caron de, Le Barbier de Séville (1772-1775), Paris, Hachette (Nouveaux Classiques), 1982, I, 1, p. 157-160.

BRUMOY, Révérend Père Pierre, Le Théâtre des Grecs, Paris, Rollin père, 1730.

CHERNI, Amor, Diderot. L'Ordre et le devenir, Genève, Droz, 2002.

DIDEROT, Denis, Le Neveu de Rameau et autres dialogues philosophiques, Paris, Gallimard (Folio), 1972. [Jean Varloot (éd.)]

DIDEROT, Denis, Jacques le Fataliste, Paris, Garnier-Flammarion, 1970. [Barbara K. Toumarkine (éd.)]

DIDEROT, Denis, «Plan d'une université pour le gouvernement de Russie », dans Euvres complètes, Paris, Garnier, 1875. [J. Assézat (éd.)]

FERRIER, Béatrice, «Pandora, ou le bonheur originel selon Voltaire », Anabases, 19, 2014, p. 313-325.

FONTENELLE, Bernard Le Bouyer de, Euvres, Paris, t. IX, 1766.

KLINGER-DOLLÉ, Anne-Hélène, « Platon latin, Platon françois : quelques traductions de la Renaissance, entre philosophie morale et fictions poétiques ", Toulouse, Université de Toulouse-Le Mirail, Séminaire PLH "Translatio : traduire et adapter les Anciens", séance du 24 III 2009, p. 1).

http://plh.univ-tlse2.fr/servlet/com.univ.collaboratif.utils.LectureFichiergw?ID...ID...

LACAN, Jacques, L'Envers de la Psychanalyse, Paris, Seuil (Champ Freudien), 1991.

LACAN, Jacques, Du discours psychanalytique, dans Lacan in Italia-Lacan en Italie, Milan, La Salamandra, 1977.

LECHEVALIER, Claire, L'Invention d'une Origine. Traduire Eschyle en France de Lefranc de Pompignan à Mazon : Le Prométhée enchaîné, Paris, Champion, 2007.

PLATON, Protagoras, Paris, Belles-Lettres, 1923. [Maurice Croiset (éd.)]

TROUSSON, Raymond, Le thème de Prométhée dans la littérature européenne, Genève, Droz, 2001.

TRUBLET. Abbé, Mémoires pour servir à l'histoire de la vie et des ouvrages de MM. de Fontenelle et de La Motte, tirés du Mercure de France, Amsterdam, Marc-M. Rey, 1761 .

UPDIKE, John, The Centaur, Harmondsworth, Penguin books, 1963. 
VOLTAIRE, Correspondence and Related Documents, Genève, Institut et musée Voltaire - Oxford, Voltaire Foundation, 1968-1978. [Théodore Besterman (éd.)]

WALTER, Éric, Jacques le Fataliste de Diderot, Paris, Hachette, 1975. 